Probability, Networks and Algorithms shared resources

M. Jonckheere, R.D. van der Mei, W. van der Weii 
Centrum voor Wiskunde en Informatica (CWI) is the national research institute for Mathematics and Computer Science. It is sponsored by the Netherlands Organisation for Scientific Research (NWO).

CWI is a founding member of ERCIM, the European Research Consortium for Informatics and Mathematics.

CWI's research has a theme-oriented structure and is grouped into four clusters. Listed below are the names of the clusters and in parentheses their acronyms.

\section{Probability, Networks and Algorithms (PNA)}

Software Engineering (SEN)

Modelling, Analysis and Simulation (MAS)

Information Systems (INS)

Copyright (C) 2007, Stichting Centrum voor Wiskunde en Informatica

P.O. Box 94079, 1090 GB Amsterdam (NL)

Kruislaan 413, 1098 SJ Amsterdam (NL)

Telephone +31205929333

Telefax +31205924199

ISSN 1386-3711 


\title{
Rate stability and output rates in queueing networks with shared resources
}

\begin{abstract}
Motivated by a variety of applications in information and communication systems, we consider queueing networks in which the service rate at each of the individual nodes depends on the state of the entire system. The asymptotic behaviour of this type of networks is fundamentally different from classical queueing networks, where the service rate at each node is usually assumed to be independent of the state of the other nodes. We study the per-node rate stability and output rates for a general class of feed-forward queueing networks with a general capacity allocation function. More specifically, we derive necessary conditions of per-node rate stability, and give bounds for the per-node output rate and asymptotic growth rates, under mild assumptions on the allocation function. For a set of parallel nodes, we further prove the convergence of the output rates and give a sharp characterization of the per-node rate stability. The results provide new intuition and fundamental insight in the stability and throughput behavior of queueing networks with shared resources.
\end{abstract}

2000 Mathematics Subject Classification: primary 60M20; 60K25, secondary 90B22.

Keywords and Phrases: queueing networks; state-dependent allocation; rate stability; output rate; growth rate 



\title{
Rate Stability and Output Rates in Queueing Networks with Shared Resources
}

\author{
M. Jonckheere ${ }^{a}$, R.D. van der Mei ${ }^{a, b}$ and ${ }^{*}$ W. van der Weij ${ }^{a}$ \\ ${ }^{a}$ CWI, Probability and Stochastic Networks, Amsterdam, The Netherlands \\ ${ }^{b}$ Vrije Universiteit, Faculty of Sciences, Amsterdam, The Netherlands
}

November 27, 2007

\begin{abstract}
Motivated by a variety of applications in information and communication systems, we consider queueing networks in which the service rate at each of the individual nodes depends on the state of the entire system. The asymptotic behaviour of this type of networks is fundamentally different from classical queueing networks, where the service rate at each node is usually assumed to be independent of the state of the other nodes. We study the per-node rate stability and output rates for a general class of feed-forward queueing networks with a general capacity allocation function. More specifically, we derive necessary conditions of per-node rate stability, and give bounds for the per-node output rate and asymptotic growth rates, under mild assumptions on the allocation function. For a set of parallel nodes, we further prove the convergence of the output rates and give a sharp characterization of the per-node rate stability. The results provide new intuition and fundamental insight in the stability and throughput behavior of queueing networks with shared resources.
\end{abstract}

\section{Key words:}

queueing networks, state-dependent allocation, rate stability, output rate, growth rate.

AMS 2000 subject classification:

primary 60M20; 60K25, secondary 90B22.

\section{Introduction}

The analysis of queueing networks has been subject to extensive research for the past few decades and has been successfully applied in many application areas. In a vast majority of papers however, it is assumed that the service rate at each of the nodes of the network is fixed. For example, in FCFS-based single- or multi-server nodes, non-idling servers are usually assumed to be autonomous entities that operate at a fixed rate, independent of the state of the other queues in the network. For the class of so-called Jackson networks [21], many stability and performance issues are well understood.

\footnotetext{
*Corresponding author. CWI, Kruislaan 413, 1098SJ Amsterdam, Netherlands. E-mail: weij@cwi.nl.
} 
In this paper, we study queueing networks in which the service rates at each of the individual nodes are not independent, but depend on the state of the entire system, according to some general capacity allocation function. For this type of models, exact structural results are rare, and fundamental insight and intuition for seemingly simple questions about stability and throughput are lacking. This motivates an in-depth study of the per-node stability for queueing networks with a general class of capacity allocation functions.

Another source of motivation stems from applications in modern computer-communication systems, in which many heterogeneous applications share parts of the available infrastructure resources. In such environments, different applications compete for access to shared resources, both at the software level (e.g., mutex and database locks, thread pools) and at the hardware level (e.g., bandwidth, processing power, disk access). For example, many Web-based services are based on multi-tiered system architectures consisting of a client tier to provide an end-user interface, a business logic tier to coordinate information retrieval and processing, and a data tier with legacy systems to store and access customer data. Each end-user initiated Web transaction typically consists of several sub-transactions that have to be processed in some fixed or probabilistic order. To this end, application servers usually implement a number of thread pools, each of which is dedicated to performing a specific sub-transaction. A particular feature of the Web server performance model proposed in $[12,23]$ is that at any moment in time the active (i.e., non-idling) threads share a common Central Processing Unit (CPU) hardware in a PS fashion. Other examples of performance models in which software resources compete for access to shared hardware resources are presented in $[14,24]$. Another interesting line of research in which the service rates among different network nodes are dependent is focused on bandwidth-sharing networks $[17,5]$, providing a natural modeling framework for describing the dynamic flow-level interaction among elastic data transfers in communication networks. Queueing models with shared resources also occur naturally in the modeling of the flow-level performance in wireline data networks where the capacity of different links are shared among competing flows [4], or in wireless networks, where a limited amount of bandwidth is shared among different users, and where customers can communicate via a cascade of intermediate hops (cf. [8]).

A considerable amount of work has been dedicated to the stability of queueing networks $[10,9$, 19, 20,22]. Controlling overload situations is essential for the design of communication networks. A well-engineered network should of course avoid to experience overload. However, the traffic fluctuations over time might lead to temporary surges that a well-designed network should deal with. A fine understanding of the behaviour of the network in overloaded is hence strongly needed. In particular, it is a fundamental issue to characterize, for given traffic conditions, which queues are going to get instable and what are the asymptotic growth rates. In particular, recent results including a sharp characterization of per-node stability for parallel nodes with a decreasing service allocation have been obtained in [7]. It clearly emerges from these papers that general results for per-node stability for multi-layered networks (or networks with bandwidth sharing) appear to be very challenging. In particular, if global stability is well known for work-conserving networks, detailed (per-node) stability remains a difficult problem. For general service allocations without monotonicity properties, it is to the best of our knowledge an open problem, even for exponentially distributed services. Instead of focusing on stochastic stability, an alternative approach to tackle stability issues is to weaken the stability definition and to investigate the so-called rate stability of the network [13]. Roughly speaking, it consists of characterizing the growth rates as linear or sub-linear. However, because in a great number of practical situations, an overload situation is characterized by a linear asymptotic per node 
growth rate, rate stability provides a precious benchmark information in cases where a more detailed stability description is almost hopeless. Using a similar line of thoughts, Egorova et al. [11] give a partial characterization of the overload behavior, for the wide class of so-called $\alpha$-fair bandwidth sharing strategies defined in [17], by examining the fluid limit by suitable scaling the number of flows in the system, and give a fixed-point equation for the corresponding asymptotic growth rates.

In this paper we consider a queueing network with Poisson arrivals, exponential service-time distributions at all nodes, internal feed-forward routing and with a structured work-conserving allocation function driving the service in all nodes, that depend on the state of the entire system. For this general model, we (1) derive necessary conditions of the per-node rate stability, and (2) give bounds for the per-node output rate. We show how to use these conditions on a two-node tandem network to get necessary and sufficient conditions of rate stability. For a set of parallel nodes with an homogeneous capacity allocation, we further prove the convergence of the output rates and give a sharp characterization of the per-node rate stability. The results provide new intuition and fundamental insight in the stability and throughput behavior of queueing networks with shared resources.

The contribution of this paper is in that respect two-fold. First, from an application point of view, intuition and understanding of the stability and throughput behavior is essential to design effective overload-control mechanisms. Second, from a theoretical point of view, the analysis of of queueing networks in which resources are shared among the different nodes raises many challenging questions regarding the stability and throughput behavior. These observations make the relevance of this paper evident.

The remainder of this paper is organized as follows. In Section 2 the model is described and the relevant notation and definitions are introduced. In particular, the difference between stochastic and rate stability is rigorously explained. In Section 3, asymptotic values as output rates and growth rates are defined. Using the structure of the considered allocation functions, important properties of these output rates are derived. In Section 4, some traffic inequalities are established leading to necessary conditions for the rate stability of each node. We then illustrate the obtained results on two toy examples. In Sections 5 and 6, we consider two special cases (i.e., the two-node tandem and the model with an arbitrary number of parallel nodes), and show that the necessary conditions derived in Sections 3 and 4 are also sufficient, under mild conditions on the capacity allocation function. Finally, in Section 6 we address a number of challenging topics for further research.

\section{$2 \quad$ odel and stability definitions}

\section{$2.1 \quad$ Network model}

We consider an open queueing network with $N$ nodes. A customer present at node $i$ is said to be of class $i(i=1, \ldots, N)$. External customers arrive at node $i$ according to a Poisson process of intensity $\lambda_{i} \geq 0$. Denote the vector of external arrival rates by $\lambda:=\left(\lambda_{1} \cdots \lambda_{N}\right)^{\top}$. The service times at node $i$ are exponentially distributed with mean $\beta_{i}=1 / \mu_{i}$. Let $\boldsymbol{\mu}:=\left(\mu_{1}, \ldots \mu_{N}\right)$. The state of the system is described by a vector $\mathbf{x}:=\left(x_{1}, \ldots, x_{N}\right)$, where $x_{i}$ represents the number of customers of class $i$. When the system is in state $\mathbf{x}$, customer of class $i$ receive a service 
rate $\phi_{i}(\mathbf{x})$, where the function $\phi(\mathbf{x}):=\left(\phi_{1}(\mathbf{x}), \ldots, \phi_{N}(\mathbf{x})\right)$ is referred to as the system capacity allocation function, for $\mathbf{x} \in \mathcal{X}:=\{0,1, \ldots,\}^{N}$. It is important to note that the various customer classes are coupled since their individual service rates may depend on the state $\mathbf{x}$ of the entire system.

Assumptions on the routing: After receiving service at node $i$, a customer is routed to node $j \in \mathcal{I}:=\{0,1, \ldots, N\}$ with probability $p_{i j}$. Denote the routing matrix by $P:=\left(p_{i j}\right)$. We adopt the convention that when $j=0$, the customer simply leaves the network. We assume that there is no loop in the routing i.e., once a customer has been served at a given node, he never returns to this node. This type of routing is often referred to as feed-forward routing. Consequently, we can order the nodes such that: $p_{i j}=0, j<i$. The routing matrix $P$ is sub-stochastic, so that $R:=\left(r_{i j}\right):=(I-P)^{-1}$ exists, where $I$ is the $N$-by- $N$ identity matrix. Moreover, let $D=\left(d_{i j}\right)$ be the $N$-by- $N$ diagonal matrix with diagonal entries, $d_{i i}:=\frac{1}{\mu_{i}}(i=1, \ldots, N)$. Using these definitions, the load offered to node $i$ is given by

$$
\rho_{i}:=\boldsymbol{\lambda}^{\top} R D \mathbf{e}_{i}=\frac{1}{\mu_{i}} \sum_{j=1}^{N} \lambda_{j} r_{j i},
$$

where $\mathbf{e}_{i}$ is the $i$-th unit vector.

Let $\mathbf{X}(t):=\left(X_{1}(t), \ldots, X_{N}(t)\right)$, where $X_{i}(t)$ denotes the number of customers at node $i$ (i.e., either waiting or being served) at time $t \geq 0$. Then the $N$-dimensional process $\{\mathbf{X}(t), t \geq 0\}$ can be described as a continuous-time Markov process with state space $\mathcal{X}$. For a subset of indexes $\mathcal{S}$, we denote $\mathbf{x}_{\mathcal{S}}$ the restriction of the vector $\mathbf{x}$ to nodes $\mathcal{S}$, i.e., $\mathbf{x}_{\mathcal{S}}=\left(x_{i}\right)_{i \in \mathcal{S}}$.

Assumptions on the service rates: Throughout the paper, the system allocation function $\phi(\mathbf{x})$ satisfies certain assumptions that we describe here.

\section{Assumption $\mathbf{A}_{1}$ (Work-conserving allocation)}

Whenever the system is not empty, all capacity is assigned to the nodes: For $\mathbf{x} \neq \mathbf{0}=(0, \ldots, 0)$,

$$
\sum_{i=1}^{N} \frac{\phi_{i}(\mathbf{x})}{\mu_{i}}=1, \quad \text { and } \phi(\mathbf{0}):=0 .
$$

Without loss of generality, the total capacity of the system is assumed to be equal to 1 in (2).

\section{Assumption $\mathbf{A}_{2}$ (Symmetric uniform limits)}

For all subset of indices $\mathcal{U} \subset\{1, \ldots, N\}$, there exists a function $g^{\mathcal{U}}$ on $\{0,1, \ldots,\}^{N-|\mathcal{U}|}$ and some strictly positive numbers $l_{i}, i \in \mathcal{U}$ such that:

$$
\forall i \in \mathcal{U}, \quad \lim _{x_{i} \rightarrow \infty, i \in \mathcal{U}} \frac{\phi_{i}(\mathbf{x})}{\mu_{i}}=l_{i} g^{\mathcal{U}}\left(\mathbf{x}_{\mathcal{S}}\right) .
$$

In many applications in computer-communication systems the allocation functions have the following structure which is a special case of work conserving allocations with symmetric uniform limits:

$$
\frac{\phi_{i}(\mathbf{x})}{\mu_{i}}=\frac{f_{i}\left(x_{i}\right)}{\sum_{j=1}^{N} f_{j}\left(x_{j}\right)}, \quad \mathbf{x} \in \mathcal{X}, \mathbf{x} \neq \mathbf{0}
$$


where $f_{i}(\cdot)$ a non-negative function such that $f_{i}(0):=0$ and $\lim _{x_{i} \rightarrow \infty} f_{i}\left(x_{i}\right)=: l_{i}<\infty(i=$ $1, \ldots, N)$. Note that in this case, Assumption 2 implies that:

$$
\forall \mathcal{U} \subset\{1, \ldots, N\}, \quad g^{\mathcal{U}}\left(\mathbf{x}_{\mathcal{S}}\right)=\sum_{i \in \mathcal{U}} l_{i}+\sum_{i \notin \mathcal{S}} f_{i}\left(x_{i}\right)^{-1} .
$$

In the sequel, we refer to these allocations as extended processor sharing allocations. Here are a few examples that have became classic in queuing theory and performance evaluation:

1. The limited processor sharing allocation defined by:

$$
f_{i}\left(x_{i}\right)=\min \left\{x_{i}, l_{i}\right\}
$$

where $l_{i}$ is a positive integer.

2. The limited discriminatory processor sharing allocation defined by:

$$
f_{i}\left(x_{i}\right)=w_{i} \min \left\{x_{i}, C_{i}\right\},
$$

where $C_{i}$ is a positive integer and $w_{i}>0$ is a weight given to class $i$. In this case $l_{i}=w_{i} C_{i}$.

3. The coupled processors allocation defined by

$$
f_{i}\left(x_{i}\right)=l_{i} 1_{x_{i}>0}
$$

where $0<l_{i}<+\infty$ is a weight associated with class $i$. In the literature, this allocation is sometimes referred to as the generalised processor sharing (GPS) allocation.

The assumptions $A_{1}$ and $A_{2}$ are not sufficient in general to get a sharp characterization of the rate stability set of the network. To get more precise results, we may assume one or both of the following conditions:

\section{Assumption $\mathbf{A}_{\mathbf{3}}$ (Asymptotic monotonicity)}

For all subset of indices $\mathcal{U} \subset\{1, \ldots, N\}$, there exists $x>0$, such that if $x_{i}>x$, for all $i \in \mathcal{U}$, then

$$
\forall i \notin \mathcal{U} \quad \frac{\phi_{i}(\mathbf{x})}{\mu_{i}} \leq l_{i} g^{\mathcal{U}}\left(\mathbf{x}_{\mathcal{S}}\right) .
$$

For extended processor sharing allocations, note that Assumption $A_{3}$ is verified in particular if:

$$
f_{i}\left(x_{i}\right) \leq l_{i} \text { for all } x_{i} \geq 0, i=1, \ldots, N .
$$

\section{Assumption $\mathbf{A}_{4}$ (Homogeneity)}

The allocation is called homogeneous if:

$$
\forall x \in \mathcal{X}, \forall \gamma \in \mathbb{R}^{+}, \phi(\gamma \mathbf{x})=\phi(\mathbf{x}) .
$$

This assumption is verified for the coupled processors allocation and for allocation based on homogeneous utility functions. For more details on bandwidth sharing networks and utilitybased allocations, we refer to [17]. 


\subsection{Stability definitions}

The study of stability of stochastic processes traditionally deals with the question of existence of a measure that is invariant to the transition operator of the process and to which the process converges in distribution or in total variation. We aim here at describing some 'per-node' stability properties, i.e. properties of the processes $\left\{X_{i}(s), s \geq 0\right\}$, for $i=1, \ldots, N$. Since the process $\left\{X_{i}(s), s \geq 0\right\}$ is not by itself a Markov process, this is generally a much more ambitious question than describing the global stability (stability of $\mathbf{X}(t)$ ) which is well known for workconserving networks (see Theorem 1 ). To the best of our knowledge, the only per-node stochastic stability results have been obtained for a set of parallel nodes with decreasing allocations and there is no such results available for the general type of networks we consider here. Because the usual definitions of stochastic stability did not lead so far (without stricter assumptions on the allocation function and the topology) to tractable results, we turn our attention to a weaker definition of stability that allows to give practical answers. We are hence primarily concerned with the property of the conservation of rates through the network. Roughly speaking, it consists of characterizing the asymptotic growing rates (rates at which the queue associated to a node builds up) as linear or sub-linear and to characterize the set of input parameters such that the incoming traffic at a node is equal to the outcoming traffic. Interesting as a first order stability property, rate stability turns out to also give useful necessary conditions of stochastic instability. For later reference, we thus define the following two notions of stability: rate stability and the stronger notion of stochastic stability.

Since we assume that the allocation function $\phi(\cdot)$ is bounded, the process $X$ is nonexplosive. Hence we may assume that $X$ and all other stochastic processes treated in the sequel have paths in the space $D=D\left(\mathcal{R}_{+}, \mathcal{Z}_{+}^{N}\right)$ of right-continuous functions from $\mathcal{R}_{+}$to $\mathcal{Z}_{+}^{N}$ with finite left limits. In the sequel, a stochastic process with paths in $D$ is viewed as a random element on the measurable space $(D, \mathcal{D})$, where $\mathcal{D}$ denotes the Borel $\sigma$-algebra generated by the standard Skorokhod topology [16].

Definition 1 (Rate stability)

The process $\left\{X_{i}(t), t \geq 0\right\}$ is said to be rate stable if

$$
\liminf _{t \rightarrow \infty} \frac{X_{i}(t)}{t}=0 \quad \text { a.s. }
$$

and the process is called rate unstable if

$$
\liminf _{t \rightarrow \infty} \frac{X_{i}(t)}{t}>0 \text { a.s. }
$$

Definition 2 (Stochastic stability)

The process $\left\{X_{i}(t), t \geq 0\right\}$ is said to be stochastically stable if

$$
\lim _{r \rightarrow \infty} \sup _{t \rightarrow \infty} \operatorname{Pr}\left\{X_{i}(t)>r\right\}=0,
$$

and the process is called stochastically unstable if

$$
\lim _{r \rightarrow \infty} \sup _{t \rightarrow \infty} \operatorname{Pr}\left\{X_{i}(t)>r\right\}>0 .
$$


Moreover, the $N$-dimensional process $\{\mathbf{X}(t), t \geq 0\}$ is said to be globally stochastically stable (or stochastically stable) if $\left\{X_{i}(t), t \geq 0\right\}$ is stochastically stable for all $i=1, \ldots, N$.

The following result, characterizing the stochastic stability of the process $\{\mathbf{X}(s), s \geq 0\}$, is well known for work-conserving networks. The total number of customers can indeed be seen as the number of customers of a single queue with unit service rate and the global stability is then a consequence of Loyne's Theorem (cf., e.g, [3]).

Theorem 1 (Global stability)

The network is globally stochastically stable if

$$
\sum \rho_{i}<1
$$

The network is globally stochastically unstable if

$$
\sum \rho_{i}>1
$$

\section{Definition 3 (Rate stability subsets)}

Let $\mathcal{S}:=\left\{i:\left\{X_{i}(t), t \geq 0\right\}\right.$ is rate stable $\}$, and $\mathcal{U}:=\left\{i:\left\{X_{i}(t), t \geq 0\right\}\right.$ is rate unstable $\}$.

Since each node is either rate stable or rate unstable, the index set $\{1, \ldots, N\}$ is partitioned into the couple $\mathcal{P}:=(\mathcal{S}, \mathcal{U})$, with $\mathcal{S} \cup \mathcal{U}=\{1, \ldots, N\}, \mathcal{S} \cap \mathcal{U}=\emptyset$. In case of rate stability, the number of customers at node $i$ grows asymptotically 'slower than $t$ ' when $t \rightarrow \infty$, at least on some trajectories. In case of stochastic stability, the process $\left\{X_{i}(t), t \geq 0\right\}$ remains in a finite neighborhood with positive probability. Remark that if $\left\{X_{i}(t), t \geq 0\right\}$ is an irreducible Markov process, then stochastic stability is equivalent to requiring $\left\{X_{i}(t), t \geq 0\right\}$ to be positive recurrent (see for example Theorem 12.25 in [16]). Note also that stochastic stability implies rate stability, as it should, but that the converse result is generally not true.

The next result underlines the relation between rate instability and stochastic instability.

\section{Proposition 1}

For $i=1, \ldots, N, \liminf _{t \rightarrow \infty} \frac{X_{i}(t)}{t}>0$, implies that $X_{i}(t) \rightarrow \infty$ in probability.

Proof: Suppose that $X_{i}(t)$ does not converge to infinity in probability. Then there exists a subsequence $\left\{t_{n}, n=0,1, \ldots\right\}$ such that $X_{i}\left(t_{n}\right) \rightarrow Z_{i}$ (in probability) for some honest (almost surely finite) random variable $Z_{i}$. Moreover, there exists another subsequence $\left\{t_{n}^{\prime}, n=0,1, \ldots\right\}$ such that $\left\{X_{i}\left(t_{n}^{\prime}\right)\right\} \rightarrow Z_{i}$ almost surely [16]. Hence, $\frac{X_{i}\left(t_{n}^{\prime}\right)-Z_{i}}{t_{n}^{\prime}} \rightarrow 0$ almost surely and since $Z_{i}$ is almost surely finite, $\frac{Z_{i}}{t_{n}^{\prime}} \rightarrow 0$ and $\frac{X_{i}\left(t_{n}^{\prime}\right)}{t_{n}^{\prime}} \rightarrow 0$ almost surely, which implies that $\liminf _{t \rightarrow \infty} \frac{X_{i}(t)}{t}=$ 0 , almost surely.

Remark 1 Many authors (see for instance [1], [13], [18]), define rate stability differently, with slightly stronger assumptions. For the purpose of our analysis, we prefer the given definition that allows to link rate instability with a convergence in probability to infinity. 


\section{Output rates and growth rates}

\subsection{Definition}

The following notation is useful in the sequel. For a given sample path $\{\mathbf{X}(s), s>0\}$, we define the Cesaro mean service rate at each node of the network by:

$$
\varphi_{i}(t)=\frac{1}{t}{ }_{0}^{t} \phi_{i}(\mathbf{X}(s)) d s, \quad i=1, \ldots, N, t>0 .
$$

The growth rate of node $i$ is defined by

$$
Y_{i}(t):=\frac{X_{i}(t)}{t}, \quad i=1, \ldots, N, t>0 .
$$

Over a given sample path $\{\mathbf{X}(s), s>0\}$, we can further define the limiting values of the mean service rate:

$$
\underline{\varphi}_{i}:=\liminf _{t \rightarrow \infty} \varphi_{i}(t), \quad \bar{\varphi}_{i}:=\limsup _{t \rightarrow \infty} \varphi_{i}(t), \quad i=1, \ldots, N,
$$

and the asymptotic growth rate of the nodes:

$$
\underline{Y}_{i}=\liminf _{t \rightarrow \infty} Y_{i}(t), \text { and } \bar{Y}_{i}=\limsup _{t \rightarrow \infty} Y_{i}(t) .
$$

From Assumption 2, the random variables $\bar{\varphi}_{i}$ are bounded, and consequently, we prove in the following section that the $\bar{Y}_{i}$ are almost surely bounded. We may therefore define the mean values of vectors, for $i=1, \ldots, N$,

$$
O_{i}:=E\left[\underline{\varphi}_{i}\right], \quad \bar{O}_{i}:=E\left[\bar{\varphi}_{i}\right], \quad Q_{i}:=E\left[\underline{Y}_{i}\right], \quad \bar{Q}_{i}:=E\left[\bar{Y}_{i}\right],
$$

and denote the corresponding vectors by $\mathbf{O}:=\left(O_{1} \cdots O_{N}\right)^{\top}, \overline{\mathbf{O}}:=\left(\bar{O}_{1} \cdots \bar{O}_{N}\right)^{\top}, \mathbf{Q}:=\left(Q_{1} \cdots Q_{N}\right)^{\top}$ and $\overline{\mathbf{Q}}:=\left(\bar{Q}_{1} \cdots \bar{Q}_{N}\right)^{\top}$. Note that rate stability of node $i$ implies that $\varphi_{i}=0$ (almost surely) and $Q_{i}=0$. Moreover, note that if node $i$ is stochastically stable, then $\bar{Q}_{i}=Q_{i}=0$ and $\bar{O}_{i}=O_{i}$.

\subsection{Properties of the asymptotic rates}

We derive here some properties of the rates of service obtained when a node is rate unstable. These properties turn out to be crucial when characterizing the rate stability of the network. It is convenient to define, for $i=1, \ldots, N$,

$$
\eta_{i}:=\mu_{i} l_{i}
$$

The next result gives a relation between the output rates and the fraction of capacity assigned for rate unstable nodes. For a given stability partitioning of the nodes $\mathcal{P}=(\mathcal{S}, \mathcal{U})$, denote

$$
\bar{Z}_{\mathcal{P}}:=E\left[\limsup _{t \rightarrow \infty} \frac{1}{t}{ }_{0}^{t} g^{\mathcal{U}}(\mathbf{X}(s)) d s\right] .
$$

Proposition 2 (Balanced output rates for rate-unstable nodes)

Assume Assumption $A_{1}$. If $i, j \in \mathcal{U}$, then

$$
\eta_{j} \bar{O}_{i}=\eta_{i} \bar{O}_{j}
$$


In particular if $l_{i}>0$ and $l_{j}>0$ :

$$
\frac{\bar{O}_{i}}{\eta_{i}}=\frac{\bar{O}_{j}}{\eta_{j}}=\bar{Z}_{\mathcal{P}}
$$

Moreover, if $\left(\alpha_{j}\right)_{j \in \mathcal{U}}$ are positive numbers, then

$$
E\left[\limsup _{t \rightarrow \infty} \sum_{j \in \mathcal{U}} \alpha_{j} \varphi_{j}(t)\right]=\sum_{j \in \mathcal{U}} \eta_{j} \alpha_{j} \bar{O}_{j} .
$$

Proof: For all $i \in \mathcal{U}, X_{i}$ converges in probability to infinity. As $\phi$ is bounded, it implies that $\frac{\phi_{i}(X(t))}{\mu_{i}}-l_{i} g^{\mathcal{U}}\left(x_{\mathcal{S}}\right) \rightarrow 0$ (in $\left.L^{1}\right)$, which gives that

$$
E\left[\frac{1}{t}{ }_{0}^{t} \frac{\phi_{i}(X(s))}{\mu_{i}}-l_{i} g^{\mathcal{U}}\left(x_{\mathcal{S}}(s)\right) d s\right] \rightarrow 0 .
$$

Using the dominated convergence theorem, we obtain that:

$$
E\left[\lim _{t \rightarrow \infty} \frac{1}{t}{ }_{0}^{t} \frac{\phi_{i}(X(s))}{\mu_{i}}-l_{i} g^{\mathcal{U}}\left(X_{\mathcal{S}}(s)\right) d s\right]=\lim _{t \rightarrow \infty} E\left[\frac{1}{t}{ }_{0}^{t} \frac{\phi_{i}(X(t))}{\mu_{i}}-l_{i} g^{\mathcal{U}}\left(x_{\mathcal{S}}\right) d s\right]=0 .
$$

We conclude by observing that:

$$
\begin{aligned}
\left.E\left[\limsup _{t \rightarrow \infty} \frac{\varphi_{i}(t)}{\mu_{i}}\right]\right]= & E\left[\lim _{t \rightarrow \infty} \frac{1}{t} \int_{0}^{t} \frac{\phi_{i}(X(s))}{\mu_{i}}-l_{i} g^{\mathcal{U}}\left(X_{\mathcal{S}}(s)\right) d s\right] \\
& +l_{i} E\left[\lim _{\sup _{t \rightarrow \infty}} \frac{1}{t} \int_{0}^{t} g^{\mathcal{U}}\left(X_{\mathcal{S}}(s)\right) d s\right] .
\end{aligned}
$$

The next two Propositions compare the outputs of rate stable and rate unstable nodes for asymptotically decreasing allocations.

Proposition 3 (Unbalanced rates between rate stable and rate unstable nodes) Assume Assumption $\left(A_{3}\right)$. Then if $i \in \mathcal{S}$ and $j \in \mathcal{U}$, it holds that

$$
\eta_{j} \bar{O}_{i} \leq \eta_{i} \bar{O}_{j}
$$

Proof: For $i \in \mathcal{S}$ and $j \in \mathcal{U}$, following the same lines as in the proof of the previous Proposition, we have

$$
\frac{\bar{O}_{i}}{\mu_{i}} \leq l_{i} E\left[\limsup _{t \rightarrow \infty} \frac{1}{t}{ }_{0}^{t} g^{\mathcal{U}}(\mathbf{X}(s))\right] d s
$$

The following Proposition uses further the structure of the extended processor sharing allocation.

\section{Proposition 4 (Comparison of output rates for different stability partitioning)}

Assume that the allocation is an extended processor sharing allocation, i.e., for $i=1, \ldots, N$,

$$
\phi_{i}(\mathbf{x})=f_{i}\left(x_{i}\right) \sum_{j=1}^{N} f_{j}\left(x_{j}{ }^{-1},\right.
$$

with $f_{i}\left(x_{i}\right) \leq l_{i}$ for all $x_{i} \geq 0, i=1, \ldots, N$, and consider two rate stability partition sets $\mathcal{P}_{1}=\left(\mathcal{S}_{1}, \mathcal{U}_{1}\right)$ and $\mathcal{P}_{2}=\left(\mathcal{S}_{2}, \mathcal{U}_{2}\right)$ such that $\mathcal{U}_{2}=\mathcal{U}_{1} \cup\{i\}=\{1, \ldots, N\}$. Then it holds that for $i=1, \ldots, N$,

$$
\eta_{j} \bar{O}_{i}^{\mathcal{P}_{1}} \leq \eta_{i} \bar{O}_{j}^{\mathcal{P}_{2}}
$$


Proof: Using again the lines of the proof of Proposition 2, we get

$$
\frac{O_{i}^{\mathcal{P}_{1}}}{\mu_{i}}=E\left[\limsup _{t \rightarrow \infty}{ }^{t} \frac{f_{i}\left(X_{i}(s)\right)}{f_{i}\left(X_{i}(s)\right)+\sum_{j \neq i} l_{j}}\right],
$$

and

$$
\frac{O_{i}^{\mathcal{P}_{2}}}{\mu_{j}}=\frac{l_{j}}{\sum_{j=1}^{N} l_{j}} .
$$

The proof then follows directly from (14) and (15) by observing that

$$
\frac{f_{i}\left(X_{i}(s)\right)}{f_{i}\left(X_{i}(s)\right)+\sum_{j \neq i} l_{j}} \leq \frac{l_{i}}{\sum_{j=1}^{N} l_{j}} .
$$

\section{$4 \quad$ ate stability necessary conditions}

\subsection{Traffic inequalities}

In the absence of stochastic stability assumptions, it is naturally not possible to define the input rate of the nodes as the solutions of the classic traffic equations as in [21] for instance. However, we can derive traffic inequalities linking the input rates and the asymptotic output rates of the network. These equations give a mathematical understanding on the common notions of mean output rates and input rates in the network.

\section{Theorem 2 (Traffic inequalities)}

The asymptotic output rates $\mathbf{O}, \overline{\mathbf{O}}$ and growth rates $\mathbf{Q}, \overline{\mathbf{Q}}$ are finite and satisfy the following linear inequalities: For $i=1, \ldots, N$,

$$
\begin{gathered}
Q_{i}+\bar{O}_{i} \leq \lambda_{i}+\sum_{j} p_{j i} \bar{O}_{j}, \\
\bar{Q}_{i}+O_{i} \geq \lambda_{i}+\sum_{j} p_{j i} O_{j} .
\end{gathered}
$$

The work conserving property brings the additional inequalities:

$$
\sum_{i=1}^{N} \frac{\bar{O}_{i}}{\mu_{i}} \geq 1, \text { and } \sum_{i=1}^{N} \frac{O_{i}}{\mu_{i}} \leq 1 .
$$

In the special case of $\rho>1$ and $\mathcal{U}=\{1, \ldots, N\}$, we have further:

$$
\sum_{i=1}^{N} \frac{\bar{O}_{i}}{\mu_{i}}=1
$$

Proof: Because of exponential service times and Poisson arrivals, $X(t)$ is a Markov process. From Assumption 2 the allocation functions $\phi_{i}($.$) , and hence the transition rates are bounded.$ 
This implies (the departure process from a node being $D_{i}(t)=A_{i}(t)-X_{i}(t)$, with $A_{i}(t)$ the arrival process at node $i$ ) that the process $\left\{M_{i}(t), t>0\right\}$, defined by

$$
M_{i}(t):=X_{i}(t)-X_{i}(0)-{ }_{0}^{t}\left\{\lambda_{i}+\sum_{j} p_{j i} \phi_{j}(\mathbf{X}(s))-\phi_{i}(\mathbf{X}(s))\right\} d s,
$$

is a martingale that satisfies $E\left[M_{i}^{2}(t)\right]<K t$ for $i=1, \ldots N, t>0$ and some $K>0$. This implies that the process $\left\{M_{i}(t) / t, t>0\right\}$ is a super-martingale bounded in $L^{2}$ and consequently, for $i=1, \ldots, N: \frac{M_{i}(t)}{t} \rightarrow 0 \quad(t \rightarrow \infty)$, a.s. Assuming for simplicity that $\mathbf{X}(\mathbf{0})=\mathbf{0}$, it is readily seen from the definitions (8) and (7) that, for $i=1, \ldots, N, t>0$,

$$
\frac{1}{t} M_{i}(t)+\lambda_{i}+\sum_{j} p_{j i} \varphi_{j}(t)-Y_{i}(t)=\varphi_{i}(t)
$$

This implies that $\lim \sup _{t \rightarrow \infty} \frac{X_{i}(t)}{t}<+\infty$ as well as

$$
\limsup _{t \rightarrow \infty} \varphi_{i}(t)=\limsup _{t \rightarrow \infty} \lambda_{i}+\sum_{j} p_{j i} \varphi_{j}(t)-Y_{i}(t) \leq \lambda_{i}+\sum_{j} p_{j i} \limsup _{t \rightarrow \infty} \varphi_{j}(t)-\liminf _{t \rightarrow \infty} Y_{i}(t) .
$$

Using the dominated convergence theorem, we get (16). (17) is obtained along the same lines. (18) follows from the dominated convergence theorem as well as the equation:

$$
1=\limsup _{t \rightarrow \infty} \sum_{i=1}^{N} \varphi_{i}(t) \leq \sum_{i=1}^{N} \limsup _{t \rightarrow \infty} \varphi_{i}(t) .
$$

If $\rho>1$, the total number of customers is transient, and hence for all $t$, almost surely $\sum_{i=1}^{N} \frac{\phi_{i}(X(t))}{\mu_{i}}=$ 1 and $\sum_{i=1}^{N} \frac{\varphi_{i}(X(t))}{\mu_{i}}=1$. The last assertion thus follows from Proposition 2.

\subsection{Necessary conditions of rate stability for converging rates}

In this subsection, we study the case $\overline{\mathbf{O}}=\mathbf{O}$, which serves as a benchmark for finding rate stability conditions in the general case. We show in the last section that we can actually prove the convergence of the asymptotic growth rates for a set parallel nodes with homogeneous, asymptotically monotone allocations.

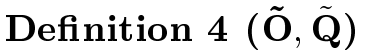

For a given stability partitioning $\mathcal{P}=(\mathcal{S}, \mathcal{U})(\mathcal{U} \neq \varnothing)$, define $(\tilde{\mathbf{O}}, \tilde{\mathbf{Q}})$ as the solution (when it exists) of:

$$
\begin{gathered}
o_{i}+q_{i}=\lambda_{i}+\sum_{j} p_{j i} o_{j}, \\
\sum_{i=1}^{N} \frac{o_{i}}{\mu_{i}}=1, \\
\frac{o_{i}}{\eta_{i}}=\frac{o_{j}}{\eta_{j}}:=\tilde{Z}_{\mathcal{P}} \quad(i, j \in \mathcal{U}), \\
q_{i}=0 \quad(i \in \mathcal{S}) .
\end{gathered}
$$


We first prove the existence of a unique solution for $\tilde{\mathbf{O}}, \tilde{\mathbf{Q}}$. We then give conditions for this solution to be positive. To simplify the notations, suppose without lost of generality that the nodes are ordered so that the stable ones are the first ones, i.e. there exists an index $m$ such that $\mathcal{S}=[1, m]$ and $\mathcal{U}=[m+1, N]$. Define $G^{\mathcal{E}_{1} \mathcal{E}_{2}}$ as the truncation of the matrix $G$ to the nodes in $\mathcal{E}_{1}, \mathcal{E}_{2}: G^{\mathcal{E}_{1} \mathcal{E}_{2}}=(G)_{i \in \mathcal{E}_{1}, j \in \mathcal{E}_{2}}$ and similarly the vector $\mathbf{v}^{\mathcal{E}}=\left(v_{i}\right)_{i \in \mathcal{E}}$. We then write the routing matrix in the following form:

$$
P=\quad \begin{array}{ll}
P^{\mathcal{S}} & P^{\mathcal{U}} \\
P^{\mathcal{U S}} & P^{\mathcal{U U}}
\end{array}
$$

Recall that the vector $\boldsymbol{\eta}$ is defined as $\boldsymbol{\eta}=\left(l_{1} \mu_{1}, \ldots, l_{N} \mu_{N}\right)$ and let us introduce the vector $\boldsymbol{\omega}^{\mathcal{S}}$, and the positive constants $\kappa_{\mathcal{P}}$ and $\chi_{\mathcal{P}}$ as:

$$
\begin{aligned}
& \omega^{\mathcal{S}}=\lambda^{\mathcal{S}} H^{\mathcal{S S}}, \\
& \kappa_{\mathcal{P}}=\sum_{i \in \mathcal{S}} \frac{\left(\eta^{\mathcal{U} P} P^{\mathcal{U S}} H^{\mathcal{S S}}\right) e_{i}}{\mu_{i}}, \quad \chi_{\mathcal{P}}=\sum_{i \in \mathcal{U}} l_{i} .
\end{aligned}
$$

where $H^{\mathcal{S S}}=\left(I-P^{\mathcal{S}}\right)^{-1}$. Remark that the matrix $H^{\mathcal{S S}}$ is not in general the restriction of the matrix $R$.

\section{Proposition 5}

Fix a partition $\mathcal{P}=(\mathcal{S}, \mathcal{U})(\mathcal{U} \neq \varnothing)$. There exists a unique solution $(\tilde{\mathbf{O}}, \tilde{\mathbf{Q}})$ of equations (20) to (23), characterized by the following equations:

$$
\begin{aligned}
\tilde{\mathbf{O}}^{\mathcal{S}} & =\left(\boldsymbol{\lambda}^{\mathcal{S}}+\tilde{Z}_{\mathcal{P}} \boldsymbol{\eta}^{\mathcal{U}} P^{\mathcal{U S}}\right) H^{\mathcal{S} \mathcal{S}}, \\
\tilde{\mathbf{O}}^{\mathcal{U}} & =\tilde{Z}_{\mathcal{P}} \boldsymbol{\eta}^{\mathcal{U}} \\
\tilde{Z}_{\mathcal{P}} & =\frac{1-\sum_{i \in \mathcal{S}} \frac{\omega_{i}^{\mathcal{S}}}{\mu_{i}}}{\kappa_{\mathcal{P}}+\chi_{\mathcal{P}}}
\end{aligned}
$$

Moreover, the solution $\tilde{\mathbf{O}}, \tilde{\mathbf{Q}}$ is positive if and only if:

$$
\begin{gathered}
\sum_{i \in \mathcal{S}} \frac{\omega_{i}^{\mathcal{S}}}{\mu_{i}} \leq 1, \\
\tilde{Z}_{\mathcal{P}} \eta^{\mathcal{U}}\left(I^{\mathcal{U U}}-P^{\mathcal{U U}}-P^{\mathcal{U S}} H^{\mathcal{S S}} P^{\mathcal{S U}}\right) \geq \lambda^{\mathcal{U}}+\lambda^{\mathcal{S}} H^{\mathcal{S S}} P^{\mathcal{S U}} .
\end{gathered}
$$

Proof: The system of equations (20) to (23)) can be rewritten as

$$
\begin{aligned}
\tilde{\mathbf{O}}^{\mathcal{S}} & =\lambda^{\mathcal{S}}+\tilde{\mathbf{O}}^{\mathcal{S}} P^{\mathcal{S S}}+\tilde{Z}_{\mathcal{P}} \boldsymbol{\eta}^{\mathcal{U}} P^{\mathcal{U S}} \\
\tilde{\mathbf{Q}}^{\mathcal{U}} & =\lambda^{\mathcal{U}}+\tilde{\mathbf{O}}^{\mathcal{S}} P^{\mathcal{S U}}+\tilde{Z}_{\mathcal{P}} \eta^{\mathcal{U}}\left(P^{\mathcal{U} \mathcal{U}}-I^{\mathcal{U} \mathcal{U}}\right), \\
\sum_{i \in \mathcal{S}} \frac{\tilde{O}_{i}^{\mathcal{S}}}{\mu_{i}} & =1-\chi_{\mathcal{P}} \tilde{Z}_{\mathcal{P}}
\end{aligned}
$$

The proposition follows from the fact that the matrices $I^{\mathcal{E}}-P^{\mathcal{E}}, \mathcal{E}=\mathcal{S S}, \mathcal{U U}$ are invertible with a positive inverse. Then, $\tilde{\mathbf{O}} \geq \mathbf{0}$ and $\tilde{Z}_{\mathcal{P}} \geq 0$, if and only if:

$$
\sum_{i \in \mathcal{S}} \frac{\omega_{i}^{\mathcal{S}}}{\mu_{i}} \leq 1 .
$$


Moreover, $\tilde{\mathbf{Q}} \geq 0$ if and only if:

$$
\tilde{Z}_{\mathcal{P}}\left(I^{\mathcal{U U}}-P^{\mathcal{U U}}\right) \eta^{\mathcal{U}} \geq \lambda^{\mathcal{U}}+\tilde{\mathbf{O}}^{\mathcal{S}} P^{\mathcal{S U}}
$$

It is remarkable that the conditions of positivity of the output rates are not sufficient to characterize the stability set. In the case of parallel nodes for instance, where we will actually derive that $\overline{\mathbf{O}}=\mathbf{O}$, we show that the additional conditions underlined in Section 3 are indeed needed to sharply characterize, for given input parameters, the rate stability set.

\subsection{Necessary conditions of rate stability}

To derive necessary conditions for a given rate stability partitioning, we bound the output rates, taking into account the assumption of a feed-forward routing. The bounds are obtained by comparing the maximum output rates with the outputs previously obtained in a (virtual) network where $\bar{O}_{i}=O_{i}, \forall i$.

\section{Lemma 1}

For $i=1, \ldots, N$, we have

$$
\bar{O}_{i} \leq \omega_{i}
$$

where the vector $\boldsymbol{\omega}=\boldsymbol{\lambda} R$ is the solution of the usual traffic equations:

$$
\boldsymbol{\omega}=\boldsymbol{\lambda}+\boldsymbol{\omega} P \text {. }
$$

Proof: Remark first that $\boldsymbol{\omega}$ exists and is unique because $R=(I-P)^{-1}$ is a well defined positive matrix since $I-P$ is substochastic. Define the degree of a node $i$ in the following way. If $\forall j=1, \ldots, N, p_{j i}=0$, then $d_{i}=0$. Otherwise, $d_{i}=\max _{j: p_{j i}>0}\left\{d_{j}\right\}$. Because of the absence of loops in the network, there exists at least one node $i_{0}$ of degree 0 (a source). Using the traffic inequalities of the previous section, we get for all nodes $i_{0}$ of degree 0 :

$$
\bar{O}_{i_{0}} \leq \lambda_{i_{0}}=\omega_{i_{0}} .
$$

We further proceed by induction on the degree of nodes. Suppose the assertion true for all degree less than $m$. Consider a node of degree $m+1$. It is receiving traffic from nodes of inferior degree. Using the traffic inequalities, the induction assumption and the definition of $\boldsymbol{\omega}$, we get:

$$
\bar{O}_{i} \leq \lambda_{i}+\sum_{j: d(j) \leq m} p_{j i} \bar{O}_{j} \leq \lambda_{i}+\sum_{j=1}^{N} p_{j i} \omega_{j}=\omega_{i} .
$$

We now derive the lemma leading to the main result of this section.

\section{Lemma 2}

For each partitioning $\mathcal{P}=(\mathcal{S}, \mathcal{U})(\mathcal{U} \neq \varnothing)$, we have:

$$
\bar{Z}_{\mathcal{P}} \geq \tilde{Z}_{\mathcal{P}}
$$

If moreover $P^{\mathcal{U S}}=0$, then

$$
\forall i \in \mathcal{S}, \bar{O}_{i} \leq \tilde{O}_{i}
$$


Note that this result holds without restriction on the routing policy, and is not limited to feedforward routing.

Proof: Using Lemma 1 and the traffic inequalities, we can write that

$$
\begin{aligned}
\overline{\mathbf{O}}^{\mathcal{S}} & \leq \boldsymbol{\omega}^{\mathcal{S}}+\bar{Z}_{\mathcal{P}} \boldsymbol{\eta}^{\mathcal{S}} P^{\mathcal{U S}} H^{\mathcal{S S}}, \text { and } \\
1 & \leq \chi_{\mathcal{P}} \bar{Z}_{\mathcal{P}}+\sum_{i \in \mathcal{S}} \frac{\bar{O}_{i}}{\mu_{i}} .
\end{aligned}
$$

Hence,

$$
\left(\chi_{\mathcal{P}}+\kappa_{\mathcal{P}}\right) \bar{Z}_{\mathcal{P}}+\sum_{i \in \mathcal{S}} \omega_{i}^{\mathcal{S}} \geq \chi_{\mathcal{P}} \bar{Z}_{\mathcal{P}}+\sum_{i \in \mathcal{S}} \frac{\bar{O}_{i}}{\mu_{i}} \geq 1
$$

which gives $\bar{Z}_{\mathcal{P}} \geq \tilde{Z}_{\mathcal{P}}$. If $P^{\mathcal{U S}}=0$, the second assertion follows from (5).

We can now derive necessary conditions for the partitioning $\mathcal{P}=(\mathcal{S}, \mathcal{U})$ to hold. We make use here of Lemma 1 and we therefore need the assumption of feed-forward routing.

\section{Theorem 3}

Suppose a given partitioning $\mathcal{P}=(\mathcal{S}, \mathcal{U})$. Then for all $i \in \mathcal{U}$ :

$$
\frac{\omega_{i}}{\eta_{i}}>\tilde{Z}_{\mathcal{P}}
$$

Proof: We write that the scaled state of an unstable node is strictly positive $\forall i \in \mathcal{U}, Q_{i}>0$, which gives, using the traffic inequalities:

$$
\forall i \in \mathcal{U}, \sum_{j=1}^{N} p_{j i} \bar{O}_{j}+\lambda_{i}-\bar{O}_{i} \geq Q_{i}>0 .
$$

Using the two previous lemmas, it leads to

$$
\forall i \in \mathcal{U}, \sum_{j=1}^{N} p_{j i} \omega_{j}+\lambda_{i}-\eta_{i} \tilde{Z}_{\mathcal{P}} \geq \sum_{j=1}^{N} p_{j i} \bar{O}_{j}+\lambda_{i}-\bar{O}_{i} \geq Q_{i}>0
$$

which gives $\frac{\omega_{i}}{\eta_{i}}>\tilde{Z}_{\mathcal{P}}$.

So far, only necessary conditions for a given rate stability partition of the nodes follow from Theorem 3. We illustrate the obtained results on two examples, where the obtained necessary conditions turn out to be sufficient in the first example and not sufficient in the second.

In the next two sections, where we derive necessary and sufficient conditions of rate stability under some of the Assumptions $A_{1}$ to $A_{4}$, for two important special cases. In Section 5 we study a two-node tandem model, and in Section 6 we consider systems of parallel nodes, with shared resources. 


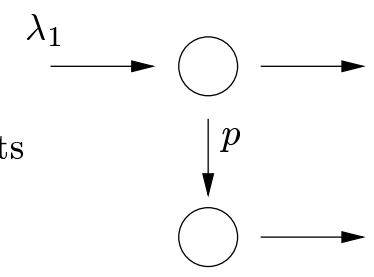

Figure 1: Two nodes in tandem.

\section{Two-node tandem model}

Consider the system of two nodes in tandem, illustrated in Figure 1 with an asymptotically decreasing extended processor sharing allocation (see Section 2). The routing matrix is given by:

$$
P=\begin{array}{ll}
0 & p \\
0 & 0
\end{array}
$$

Thus, a fraction $p$ of the output rate of the first node is sent as input rate to the second node. The following traffic equations and inequalities hold (Theorem 2):

$$
\begin{aligned}
& Q_{1}+\bar{O}_{1}=\lambda_{1}, \\
& Q_{2}+\bar{O}_{2} \leq p \bar{O}_{1}, \\
& \bar{O}_{1}+\bar{O}_{2} \geq 1 .
\end{aligned}
$$

For the corresponding virtual model verifying $\overline{\mathbf{O}}=\mathbf{O}$, the traffic equations are:

$$
\begin{aligned}
\tilde{O}_{1}^{\mathcal{P}} & =\lambda_{1}-\tilde{Q}_{1}^{\mathcal{P}}, \\
\tilde{O}_{2}^{\mathcal{P}} & =p \tilde{O}_{1}^{\mathcal{P}}-\tilde{Q}_{2}^{\mathcal{P}}, \\
\frac{\tilde{O}_{1}^{\mathcal{P}}}{\mu_{1}} & +\frac{\tilde{O}_{2}^{\mathcal{P}}}{\mu_{2}}=1, \\
\frac{\tilde{O}_{i}^{\mathcal{P}}}{\eta_{i}} & =\tilde{Z}_{\mathcal{P}} \text { for } i \in U .
\end{aligned}
$$

By $\mathcal{P}$ we denote the partition of nodes according to their rate stability. $\mathcal{P}$ can thus be $(\mathcal{S}, \mathcal{S})$, $(\mathcal{U}, \mathcal{S}),(\mathcal{S}, \mathcal{U})$, and $(\mathcal{U}, \mathcal{U})$. The solution of $\tilde{O}$ and $\tilde{Q}$ are given in Table 5 for each stability subset $\mathcal{P}$. According to Theorem 3 the network is globally stochastically stable if and only if $\rho<1$

\begin{tabular}{ccccc}
$\mathcal{P}$ & $\tilde{Q}_{1}$ & $\tilde{Q}_{2}$ & $\tilde{O}_{1}$ & $\tilde{O}_{2}$ \\
\hline$(\mathcal{S}, \mathcal{S})$ & 0 & 0 & $\lambda_{1}$ & $\lambda_{1} p$ \\
$(\mathcal{S}, \mathcal{U})$ & 0 & $p \lambda_{1}-\left(1-\frac{\lambda_{1}}{\mu_{1}}\right) \mu_{2}$ & $\lambda_{1}$ & $\left(1-\frac{\lambda_{1}}{\mu_{1}}\right) \mu_{2}$ \\
$(\mathcal{U}, \mathcal{S})$ & $\lambda_{1}-\frac{\mu_{1} \mu_{2}}{p \mu_{1}+\mu_{2}}$ & 0 & $\frac{\mu_{1} \mu_{2}}{p \mu_{1}+\mu_{2}}$ & $\frac{p \mu_{1} \mu_{2}}{p \mu_{1}+\mu_{2}}$ \\
$(\mathcal{U}, \mathcal{U})$ & $\lambda_{1}-\frac{l_{1} \mu_{1}}{l_{1}+l_{2}}$ & $\frac{p l_{1} \mu_{1}}{l_{1}+l_{2}}-\frac{l_{2} \mu_{2}}{l_{1}+l_{2}}$ & $\frac{l_{1} \mu_{1}}{l_{1}+l_{2}}$ & $\frac{l_{2} \mu_{2}}{l_{1}+l_{2}}$
\end{tabular}

Table 1: Output rates for the stability subsets. 
which writes

$$
\lambda_{1}<\frac{\mu_{1} \mu_{2}}{p \mu_{1}+\mu_{2}} .
$$

Note that in this case $\overline{\mathbf{O}}=\tilde{\mathbf{O}}=\mathbf{O}$ and $\overline{\mathbf{Q}}=\tilde{\mathbf{Q}}=\mathbf{Q}$.

Necessary conditions for $(\mathcal{U}, \mathcal{U}):$ For the partition $(\mathcal{U}, \mathcal{U})$, given that $\tilde{Z}_{\mathcal{P}}=\frac{1}{l_{1}+l_{2}} \boldsymbol{\omega}=$ $\left(\lambda_{1}, p \lambda_{1}\right)$, the following conditions given by Theorem 3 are necessary for the partition $(\mathcal{U}, \mathcal{U})$ :

$$
\begin{aligned}
p & >\frac{l_{2} \mu_{2}}{l_{1} \mu_{1}} \\
\lambda_{1} & >\frac{\mu_{1} l_{1}}{l_{1}+l_{2}} .
\end{aligned}
$$

Using the last assertion in Theorem 2, we further obtain that $\bar{Z}_{\mathcal{P}}=\tilde{Z}_{\mathcal{P}}$.

Necessary conditions for $(\mathcal{S}, \mathcal{U})$ : For the partitions $(\mathcal{U}, \mathcal{S})$ and $(\mathcal{S}, \mathcal{U})$, the necessary conditions raised by Theorem 3 lead to the already known condition $\rho>1$. Using Theorem 3 $\left(\bar{Z}_{\mathcal{P}}>\tilde{Z}_{\mathcal{P}}\right)$, the first traffic equation and the additional inequalities given by 2 and Proposition 3, we obtain:

$$
\frac{\lambda_{1}}{l_{1} \mu_{1}}=\frac{\tilde{O}_{1}^{(\mathcal{S}, \mathcal{U})}}{\eta_{1}}=\frac{\bar{O}_{1}^{(\mathcal{S}, \mathcal{U})}}{\eta_{1}}<\frac{\bar{O}_{1}^{(\mathcal{U}, \mathcal{U})}}{\eta_{1}}=\frac{\tilde{O}_{1}^{(\mathcal{U}, \mathcal{U})}}{\eta_{1}}=\frac{1}{l_{1}+l_{2}},
$$

which leads to the necessary equation $\lambda_{1}<\frac{\mu_{1} l_{1}}{l_{1}+l_{2}}$.

Necessary conditions for $(\mathcal{U}, \mathcal{S})$ :

$$
\frac{\mu_{1} p \mu_{2}}{\left(\mu_{1} p+\mu_{2}\right) l_{2} \mu_{2}}=\frac{\tilde{O}_{2}^{(\mathcal{U}, \mathcal{S})}}{\eta_{2}} \leq \frac{\bar{O}_{2}^{(\mathcal{U}, \mathcal{S})}}{\eta_{2}}<\frac{\bar{O}_{2}^{(\mathcal{U}, \mathcal{U})}}{\eta_{2}}=\frac{\tilde{O}_{2}^{(\mathcal{U}, \mathcal{U})}}{\eta_{2}}=\frac{1}{l_{1}+l_{2}},
$$

and this leads to the necessary inequality that $p<\frac{l_{2} \mu_{2}}{l_{1} \mu_{1}}$.

The obtained necessary conditions are easily seen to lead to a complete partitioning of the parameter set, which gives a sharp characterization of the stability set. As a consequence, the obtained conditions are both necessary and sufficient, except on a boundary set of input parameters.

In Figure 2, the stability set is pictured for two different sets of input parameters.

\section{Parallel nodes}

In this section, we consider parallel nodes and thus suppose that there is no internal routing, i.e., $p_{i j}=0$, for all $i, j$. In that case, we can derive a sharp characterization of the per-node rate stability. To this end, we first show that in that case, the traffic inequalities are actually a set of traffic equations (Theorem 4). This allows to prove that the output rates and asymptotic growth rates are converging. Using the results of Sections 3 and 4, we then derive a characterization of the per-node stability (Theorem 5). 

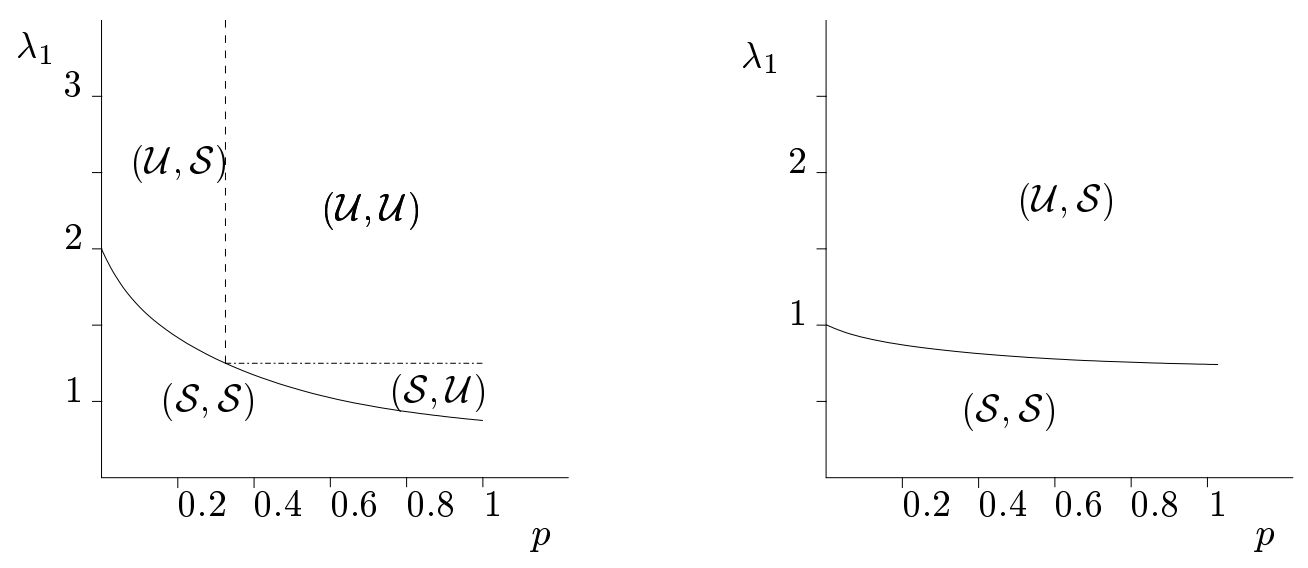

Figure 2: Stability regions with $\left(\mu_{1}, l_{1}, \mu_{2}, l_{2}\right)=(3,1,1,1)$ for the left figure, and $\left(\mu_{1}, l_{1}, \mu_{2}, l_{2}\right)=$ $(1,1,3,1)$ for the right figure.

\subsection{Extended traffic equations}

In this subsection, we precise the traffic inequalities obtained in the general case by deriving traffic equations linking the input rates and the asymptotic output rates of the network.

\section{Theorem 4 (Extended traffic equations)}

The asymptotic output rates $\mathbf{O}, \overline{\mathbf{O}}$ and growth rates $\mathbf{Q}, \overline{\mathbf{Q}}$ are finite and satisfy the following linear equations: For $i=1, \ldots, N$,

$$
\begin{aligned}
Q_{j}+\bar{O}_{i} & =\lambda_{i}, \\
\bar{Q}_{j}+O_{i} & =\lambda_{i} .
\end{aligned}
$$

Proof: We follow the same lines as in Theorem 2,

$$
M_{i}(t):=X_{i}(t)-X_{i}(0)-{ }_{0}^{t}\left\{\lambda_{i}-\phi_{i}(\mathbf{X}(s))\right\} d s,
$$

is a martingale that satisfies $E\left[M_{i}^{2}(t)\right]<K t$ for $i=1, \ldots N, t>0$ and some $K>0$. This implies that $\lim \sup _{t \rightarrow \infty} \frac{X_{i}(t)}{t}<+\infty$ and $\liminf _{t \rightarrow \infty} Y_{i}(t)=\lambda_{i}-\lim \sup _{t \rightarrow \infty} \varphi_{i}(t)$. Using the dominated convergence theorem, we get equation (27) and (28).

\subsection{Output rates convergence}

We fix $\mathcal{P}$ a partition of nodes such that nodes in $\mathcal{S}$ are rate stable while nodes in $\mathcal{U}$ are rate unstable. In the following proposition, we prove that the output rates of the different nodes converge which further allows a complete description of the rate stability set.

\section{Proposition 6}

Consider a set of parallel nodes with a decreasing allocation verifying the assumptions $A_{1}, A_{2}$ and $A_{4}$. Then,

$$
\begin{aligned}
\frac{X_{i}(t)}{t} & \rightarrow Q_{i}, \text { in probability }, \\
\varphi_{i}(t) & \rightarrow O_{i}, \text { in probability },
\end{aligned}
$$


with

$$
\begin{gathered}
\tilde{O}_{i}=\lambda_{i} \quad(i \in \mathcal{S}), \quad \tilde{O}_{i}=\tilde{Z}_{\mathcal{P}} \eta_{i} \quad(i \in \mathcal{U}), \\
\tilde{Q}_{i}=0 \quad(i \in \mathcal{S}), \quad \tilde{Q}_{i}=\lambda_{i}-\tilde{Z}_{\mathcal{P}} \eta_{i} \quad(i \in \mathcal{U}) .
\end{gathered}
$$

where

$$
\tilde{Z}_{\mathcal{P}}:=\frac{1-\sum_{j \in \mathcal{S}} \frac{\lambda_{j}}{\mu_{j}}}{\sum_{j \in \mathcal{U}} l_{j}}=\frac{1-\sum_{j \in \mathcal{S}} \rho_{j}}{\chi_{\mathcal{P}}} .
$$

Proof: Let us first prove the convergence of the rates. Note that an asymptotically monotone homogeneous allocation is actually monotone. Using the homogeneity and the monotony of the allocation, we get that for $t$ large and for $i=1, \ldots, N$ :

$$
\phi_{i}(\mathbf{X}(t))=\phi_{i} \frac{\mathbf{X}(t)}{t} \geq \phi_{i}(\overline{\mathbf{Q}}),
$$

which implies

$$
\varphi_{i}(t) \geq \phi_{i}(\overline{\mathbf{Q}}) .
$$

This leads to:

$$
\bar{Q}_{i} \leq \lambda_{i}-\phi_{i}(\overline{\mathbf{Q}}) \quad i=1, \ldots, N
$$

Similarly, for $i \in \mathcal{U}$ :

$$
Q_{i} \geq \lambda_{i}-\phi_{i}(\mathbf{Q}), \quad i=1, \ldots, N .
$$

Summing these inequalities for $i=1, \ldots, N$ and using the property of a work conserving allocation, we obtain that:

$$
\sum_{i=1}^{N} \frac{Q_{i}}{\mu_{i}} \geq \sum_{i=1}^{N} \frac{\lambda_{i}}{\mu_{i}}-1 \geq \sum_{i=1}^{N} \frac{\bar{Q}_{i}}{\mu_{i}} .
$$

We hence deduce that $\bar{Q}_{i}=Q_{i}$ and as a consequence:

$$
i=1, \ldots, N, \quad \bar{O}_{i}=O_{i}=\tilde{O}_{i} .
$$

The convergence in $L^{1}$ of $\varphi_{i}(t)$ to a constant imply the convergence in probability of $\varphi_{i}(t)$ which combined with the almost sure convergence of the difference $Y_{i}(t)-\varphi_{i}(t)$ imply the convergence of $Y_{i}(t)$ in probability. The traffic equations defined previously together with the system 4.2 allow us to complete the proof.

Remark 2 It appears plausible to prove an almost sure convergence for these processes even without the assumption of exponential service times nor Poisson arrivals. This result is out of the scope of this paper but we refer to the method presented in [15] and further used for a set of discriminatory processor sharing nodes (DPS) in [2] for such a derivation. These techniques, jointly used with the traffic conservation used here would prove the stated convergence in the context of stationary marked point processes. 


\subsection{Characterization of the per-node rate stability}

We assume without loss of generality that the nodes are ranked in decreasing order of the loads $\zeta_{i}:=\frac{\lambda_{i}}{l_{i} \mu_{i}}$, in the sense that

$$
\zeta_{1} \leq \cdots \leq \zeta_{N}
$$

The following result shows the relation between the ordering of the nodes and the per-node rate stability.

\section{Proposition 7}

If node- $i$ is rate stable and $j<i$, then node $j$ is also rate stable.

Proof: Suppose $j \in \mathcal{U}, i \in \mathcal{S}$ and $j<i$. From Proposition 3, we get: $\frac{\bar{O}_{i}}{\eta_{i}}<\frac{\bar{O}_{j}}{\eta_{j}}$. From Theorem 3 , it follows that $\bar{O}_{i}=\lambda_{i}$ and from Theorem $3, \bar{O}_{j} \leq \lambda_{j}$. We thus find that

$$
\zeta_{i}=\frac{\bar{O}_{i}}{\eta_{i}}<\frac{O_{j}}{\eta_{j}} \leq \frac{\lambda_{j}}{\eta_{j}}
$$

This contradicts $\zeta_{j} \leq \zeta_{i}$.

Denote $\tilde{Z}(m)=\tilde{Z}_{\{1 \ldots, m\}}=\frac{\left(1-\sum_{i<m} \rho_{i}\right)}{\sum_{i>m} l_{i}}$ The following result shows that the partitioning $\mathcal{P}=$ $(\mathcal{S}, \mathcal{U})$ has a simple structure.

\section{Theorem 5 (Structure of stability partitioning)}

Consider a set of parallel nodes with a decreasing allocation verifying the assumptions $A_{1}, A_{2}$ and $A_{4}$. The stability partitioning $\mathcal{P}=(\mathcal{S}, \mathcal{U})$ is characterized as follows:

$\mathcal{P}=(\mathcal{S}, \mathcal{U})$ with $\mathcal{S}=\{1 \ldots, m\}$ and $\mathcal{U}=\{m+1, \ldots N\}$ if and only if

$$
\zeta_{m} \leq \tilde{Z}(m)<\zeta_{m+1}
$$

Proof: Using Proposition 7, there exists $k$ such that $\mathcal{S}=\{1 \ldots, k\}$ and $\mathcal{U}=\{k+1, \ldots N\}$. Theorem 3 combined with Proposition 6 gives that $\tilde{Z}(k)=\bar{Z}(k)<\zeta(k+1)$. Proposition 7 gives:

$$
\frac{\bar{O}_{k}}{\eta_{k}} \leq \frac{\bar{O}_{k+1}}{\eta_{k+1}}
$$

which combined with the traffic equations leads to

$$
\zeta_{k} \leq \tilde{Z}(k) .
$$

As $\tilde{Z}(\cdot)$ is a decreasing function, we conclude that $m=k$.

We emphasize that Theorem 5 gives a complete characterization of the rate stability partitioning for model instances that satisfy Assumptions $A_{1}, A_{2}$ and $A_{4}$ and are monotone. Typical examples of such allocations are the coupled processors allocation (defined in Section 2.1), and for some utility-based allocation on some tree topology (see [6]). 


\section{Concluding remarks and challenges for further research}

The results presented in this paper provide new intuition and fundamental insight in the stability and throughput behavior of queueing models in which resources are shared among different queues. These results should be viewed as a first step in understanding the behavior of this type of queueing networks, and open up a wealth of challenging open research questions. Some of these questions will be briefly touched upon below.

In the context of stability and throughput characteristics, several interesting questions remain to be answered. First, when $X$ is a continuous-time Markov chain, it actually remains an open and crucial question to know for which input parameters, rate instability of node $i$ coincides to the convergence of $X_{i}$ to infinity (either in probability or in law). In [7], per-node stochastic stability is established for parallel nodes with monotone allocation functions. It is remarkable that, except possibly on the boundary of the stability sets, the conditions of rate instability (and thus stochastic instability) that we have derived here coincide with the sharp characterization of the stochastic instability set given in [7]. This encouraging observation calls for a generalization of this result to more complex topologies. Second, the derivation of necessary conditions for rate stability for models that are not covered by the ones discussed in Sections 5 and 6 is an open area. For example, consider a seemingly simple three-node network where all customers arrive at node 1 , and then either move to node 2 (with probability $p_{1}$ ) or to node 3 (with probability $p_{2}$ ) before departing from the system, with $0 \leq p_{1}+p_{2}<1$. Then it can be shown that the necessary conditions obtained in this paper do not lead to a full partitioning of the parameter set. This observation shows that extension of the necessary conditions presented in Sections 3 and 4 to a broader class of models is far from trivial, and addresses an open area for further research. In addition to considering stability and throughput, one may also be interested other performance metrics such as steady-state sojourn-time distributions of customers at the different nodes, the optimal static or dynamic assignment of servers to the nodes, depending on the state of the system. Derivation of such results is another interesting topic for further research.

\section{eferences}

[1] E. Altman, S.G. Foss, E.R. Riehl, , and S. Stidham Jr. Performance bounds and pathwise stability for genrealized vacation and polling systems. Technical Report UNC/OR TR93-8, Department of Operations Research, University of North Carolina at Chapel Hill, 1993.

[2] E. Altman, T. Jiménez, and D. Kofman. Dps queues with stationary ergodic service times and the performance of tcp in overload. In Proceedings of IEEE Infocom, Hong Kong, 2004.

[3] F. Baccelli and P. Bremaud. Elements of Queueing Theory. Palm-Martingale Calculus and Stochastic Recurrences. Springer-Verlag, New York Inc, 1991.

[4] T. Bonald and L. Massoulié. Impact of fairness on Internet performance. In Proc. ACM Sigmetrics / Performance 2001, 2001.

[5] T. Bonald, L. Massoulié, A. Proutière, and J. Virtamo. A queueing analysis of max-min fairness, proportional fairness and balanced fairness. Queueing Syst., 53(1-2):65-84, 2006.

[6] T. Bonald and A. Proutière. On stochastic bounds for monotonic processor sharing networks. Queueing Syst., 47:81-106, 2004. 
[7] S.C. Borst, M. Jonckheere, and L. Leskelä. Stability of parallel queueing systems with coupled service rates. to appear in Discrete Events and Stochastic Systems, 2007.

[8] T. Coenen, H. van den Berg, and R.J. Boucherie. A flow level model for wireless multihop ad hoc networks throughput. In Proceedings 3rd international working conference on Performance Modelling and Evaluation of Heterogeneous Networks, HETNETS, number P34, Ilkley, England, 2005.

[9] J.G. Dai. On positive Harris recurrence of multiclass queueing networks: A unified approach via fluid limit models. Ann. Appl. Probab., 5(1):49-77, 1995.

[10] G. de Veciana, T.J. Lee, and T. Konstantopoulos. Stability and performance analysis of networks supporting elastic services. IEEE/ACM Trans. Netw., 9(1):2-14, 2001.

[11] R. Egorova, S.C. Borst, and A.P. Zwart. Bandwidth sharing networks in overload. Performance Evaluation, 64:978-993, 2007.

[12] W.K. Ehrlich, R. Hariharan, P.K. Reeser, and R.D. van der Mei. Performance of web servers in a distributed computing environment. In Teletraffic Engineering in the Internet Era, proceedings ITC, volume 17, pages 137-148, Salvador de Bahia, Brazil, 2001.

[13] M. El-Taha and S. Stidham. Sample-path stability conditions for multiserver input-output processes. Journal of Applied Mathematics and Stochastic Analysis, 7:437-456, 1994.

[14] M. Harkema, B.M.M. Gijsen, R.D. van der Mei, and Y. Hoekstra. Middleware performance modelling. In Proceedings international Symposium on Performance Evaluation of Computer and Telecommunication Systems, SPECTS, pages 733-742, San Jose, CA, 2004.

[15] A. Jean-Marie and P. Robert. On the transient behavior of the processor sharing queue. Queuing Systems, 17:129-136, 1994.

[16] O. Kallenberg. Foundations of Modern Probability. Springer, second edition, 2002.

[17] L. Massoulie and J.W. Roberts. Bandwidth sharing: objectives and algorithms. In Proc. IEEE Infocom 1999, pages 1395-1403, 1999.

[18] R. Mazumdar, F. Guillemin, V. Badrinath, and R. Kannurpatti. On pathwise behavior of queues. Oper. Res. Let., 12:263-270, 1992.

[19] S.P. Meyn. Transcience of multiclass queueing networks with via fluid limit models. Ann. Appl. Probab., 5(4):946-957, 1995.

[20] R.R. Rao and A. Ephremides. On the stability of interacting queues in a multiple-access system. IEEE Trans. Inf. Theory, 34(5), 1988.

[21] R. Serfozo. Introduction to Stochastic Networks. Springer, 1999.

[22] W. Szpankowski. Stability conditions for some distributed systems: buffered random access systems. Adv. Appl. Probab., 26(2):498-515, 1994.

[23] R.D. van der Mei, W.K. Ehrlich, P.K. Reeser, and J.P. Francisco. A decision support system for tuning web servers in distributed object-oriented network architectures. $A C M$ Performance Evaluation Review, 27:57-62, 2000. 
[24] R.D. van der Mei, R. Hariharan, and P.K. Reeser. Web server performance modeling. Telecommunication Systems, 16:361-378, 2001. 